Cahiers $d u$ MONDE RUSSE

\section{Cahiers du monde russe}

Russie - Empire russe - Union soviétique et États indépendants

61/3-4| 2020

Écritures visuelles, sonores et textuelles de la justice

Olivier CAMY, Cécile PICHON-BONIN, La politique de Malévitch

\title{
Emanuel Landolt
}

\section{OpenEdition}

Journals

Édition électronique

URL : https://journals.openedition.org/monderusse/12219

DOI : $10.4000 /$ monderusse. 12219

ISSN : 1777-5388

Éditeur

Éditions de l'EHESS

Édition imprimée

Date de publication : 1 juillet 2020

Pagination : $553-556$

ISBN : 978-2-7132-2832-2

ISSN : $1252-6576$

Référence électronique

Emanuel Landolt, "Olivier CAMY, Cécile PICHON-BONIN, La politique de Malévitch », Cahiers du monde russe [En ligne], 61/3-4 | 2020, mis en ligne le 01 juillet 2020, consulté le 14 septembre 2022. URL http://journals.openedition.org/monderusse/12219; DOI : https://doi.org/10.4000/monderusse. 12219 


\section{Olivier CAMY, Cécile PICHON-BONIN}

\section{La politique de Malévitch}

Tusson : Édition du Lérot, 2019, 168 p.

Cet ensemble de contributions est issu d'un colloque international qui s'est tenu à Dijon les 6-7 décembre 2018 ayant pour titre "La politique de Malévitch ». Ce titre, qui semble immédiatement suggérer au lecteur qu'il existe une politique malévitchienne, ne rend pas justice à la tentative des différentes contributions de problématiser la complexité et le caractère énigmatique du positionnement politique de Malevič : en effet celui-ci est difficilement saisissable, tant sur le plan géographique, institutionnel, artistique que personnel. Quelques notes non-publiées mises en lumière par Šatskih avaient par ailleurs révélé un Malevič éminemment sceptique et critique vis-à-vis du régime. Une lecture entre les lignes au sens de Léo Strauss ${ }^{1}$ s'impose donc. Par ailleurs, le caractère théorique, pédagogique et philosophique des recherches de Malevič ajoute un double fond interprétatif non négligeable. Sont donc ici rassemblés une série d'articles qui permettent de traduire un peu mieux la complexité du rapport que Malévith entretint avec la politique, cela sur trois plans : pratique, théorique et artistique.

Le recueil s'ouvre sur un court texte d'Olivier Camy qui cherche à montrer l'actualité de Malevič en traçant des liens entre sa pensée et celles de Baudrillard, d'Husserl ou encore de Rancière, produisant plus de confusion que de clarification 
sur ce dont la politique de Malevič est le nom. Il est dommage par conséquent que la politique n'ait pas été mieux problématisée en exergue, sachant que celle-ci se situe à différents niveaux, sa charge sémantique ou sa définition étant amenée à changer selon que Malevič parle en son nom d'artiste, de fonctionnaire ou d'enseignant. Par ailleurs, la politique au sein des avant-gardes, à savoir la lutte souvent concurrentielle au sein du champ artistique, de la culture picturale de son temps aurait pu constituer un terrain pour problématiser cette même notion.

Le premier article, de Christiane Post, retrace chronologiquement l'engagement de Malevič dans la politique des musées de l'immédiate après-révolution à la période de Vitebsk, montrant très bien combien celui-ci reposait sur une défense des droits inaliénables de l'artiste. Cet engagement se retrouve jusque dans l'activité du peintre au sein des musées, autant dans leur organisation, dans l'acquisition d'œuvres que dans la conception de l'accrochage. Cet activisme a été essentiel dans la création des premiers musées d'art contemporain, plus d'une trentaine en 1921 (Malevič n'est pas le seul et il faudrait mentionner ici également la lutte sur le terrain de l'expérimentation muséologique entre Kandinskij et Rodčenko, finement analysée par Maria Gough), mais également dans les activités d'enseignement de Malevič, cela dans des conditions extrêmement précaires. La politique institutionnelle et pédagogique de Malevič ne s'arrête pas à Vitebsk et à Petrograd. La contribution de Tetyana Filevska permet au lecteur francophone de découvrir le fascinant volet ukrainien de la trajectoire de Malevič, un terrain que Jean-Claude Marcadé arpente depuis bien des années et qu'une récente publication à Kiev permet d'apprécier ${ }^{2}$. Au cours des années 1928-1930, Malevič enseigne à l'institut d'art de Kiev et publie une douzaine d'articles dans la revue ukrainienne Nova Generacija de nombreux articles. Si les archives concernant son enseignement ont malheureusement disparu, la découverte d'archives privées en 2015 auprès de Marian Kropyvnyckyi, assistant personnel de Malevič, permet de révéler les manuscrits d'articles parfois sensiblement modifiés à leur publication. Le texte donne à voir l'essor de ce que l'on a appelé le « Bauhaus de Kiev » et d'inscrire l'activité pédagogique que Malevič y a menée dans la continuité de ses expériences précédentes.

L'article très fouillé d'Elitza Dulguerova examine la place essentielle qu'occupe l'espace au-delà du tableau chez Malevič et les régions expérientielles que celui-ci réalise. Sont successivement examinées les explorations de l'espace dans l'œuvre puis dans l'exposition. L'auteure montre combien la complexité de l'espace de l'œuvre, puis l'accrochage révèlent un espace concret, continu, un espace qui fonctionne littéralement comme force agissante. Une notion clé pour comprendre comment théorie et pratique se complètent et se renforcent chez Malevič, mais une notion clé aussi pour l'avant-garde russe en général. Cette force possède un champ d'action qui va littéralement contaminer les réflexions sur différents médiums abordées par Malevič : affiche, photographie, architecture, montrant comment, en particulier dans l'architecture, l'espace renouvelé, repensé, acquiert la vigueur d'une expérience et d'une sensation ; l'auteur montre ici très bien tout ce qui sépare le suprématisme du constructivisme précisément sur ce point. 
Dans la deuxième partie, Olivier Camy offre un long article sur les relations entre esthétique et politique chez Malevič. Une question éminemment complexe dont l'auteur cherche à montrer qu'elle est tributaire d'une lecture de la manière dont Malevič s'est lui-même situé et compris, tant le peintre a été soumis à la censure et probablement à l'autocensure. Dans un travail critique par rapport aux travaux de Martineau qui rapprochait Malevič de Heidegger, l'auteur essaie de démontrer combien la pensée philosophique de Malevič est tributaire d'une ontothéologie, l'inscrivant même dans « une tradition néo-platonicienne remaniée par la spiritualité russe ». Voyant dans les spéculations du texte « Dieu n'est pas détrôné » une forme de théologie négative, l'auteur tente une traduction politique de ces termes par l'ontologie spécifique qu'elle apporterait à la notion de révolution, à savoir l'être-excitation éprouvé au sein d'une communauté sans intermédiaire institutionnel. Une expérience entièrement fondée sur la sensation. La politique malévitchienne est ainsi ramenée ontologiquement à l'expérience du sans-objet. Olivier Camy cherche encore à montrer en conclusion combien cette ontologie politique est inédite en ce qu'elle se propose d'aller plus loin que la théorie marxiste de l'aliénation économique faisant de la transformation de notre rapport au monde un horizon d'émancipation.

Jean-Claude Marcadé examine de son côté le rapport de Malevič à l'anarchisme. Si le peintre ne se revendique pas d'une tradition anarchiste en particulier, il faudrait plutôt parler d'une idée diffuse qui se rapprocherait de différentes doctrines. Plusieurs éléments sont ainsi identifiés: l'acceptation de la révolution comme rupture avec le monde ancien, la célébration de la destruction et la primauté du Moi. L'originalité de l'article réside également dans le fait que l'auteur considère la question de l'anarchisme dans le cadre du rapport critique qu'entretient le peintre avec les dérives totalitaires (notamment par le filtre de l'identité ukrainienne de l'artiste), celui-ci étant plus soucieux de faire coïncider sa vision théorico-poiétique avec la révolution soviétique que de faire de la politique, développant un subtil « langage de camouflage » pour masquer sa critique des dérives totalitaires. Jean-Claude Marcadé cite encore Malevič pour appuyer cette revendication radicale d'autonomie : «L'artiste est une personne libérée de tout ce qui n'est pas construit picturalement ». Aage Hansen Löve dresse le portrait d'un artiste lucide et autocritique et explore également cette pensée anarchiste nébuleuse, à la fois critique du communisme comme nouvelle religion et du culte de la personnalité.

Sur le plan de la théorie de l'image se niche également une dimension politique toutefois difficilement assimilable à l'anarchisme en raison de sa composition religieuse. Dans une approche centrée autour de la notion de lik (face, visage), Ioulia Podoroga cherche à montrer comment cette notion de visage fonctionne à la fois comme critique des illusions de l'image comme représentation et comme point de passage vers le monde sans-objet selon un schéma dialectique tripartite : diversité des visages, face unique, négation de la face. L'auteur situe la pleine réalisation de ce modèle ultime de face dans la dernière période suprématiste, à équidistance du pôle théologique et révolutionnaire. Sainteté d'une face sans visage, sans image, 
les dernières figures paysannes révèlent « une nouvelle image de l'homme » et indiquent les voies d'émancipation pour l'humanité.

Les deux articles qui viennent clore le volume se penchent sur la période tardive de Malevič. Thyago Marão Villela s'intéresse à l'éclectisme de cette période, mêlant figuration et allusions aux différentes cultures picturales traversées par Malevič. L'auteur identifie ce regard en arrière comme politique. Y préside une logique citationnelle et de réécriture du passé artistique russe comme forme de résistance critique à l'incessante projection vers l'avenir de la doxa stalinienne. L'auteur trouve ensuite un appui dans les thèses de Benjamin sur l'histoire pour penser cette résistance à l'impératif de progrès. Le présent soviétique au lieu d'engendrer un présent, se retrouve hanté par un passé qui le remplit entièrement. Sebastian Egenhofer saisit lui un motif constant de l'approche malévitchienne, des premières toiles impressionnistes aux travaux tardifs, celui du sans-objet. Un sans-objet conçu comme « l'état dynamique et énergétique du réel » avant sa solidification dans le monde objectif. Ce réel à l'état pur est porteur d'une économie de l'abondance, que l'auteur oppose à la lutte contre l'inertie du constructivisme qu'il identifie très justement à une approche contrôlante (planifiée) gouvernée par la loi du manque. À cette abondance répond précisément un changement de motif dans la dernière partie de l'œuvre, subtilement relevé par l'auteur. Le rejet de la perspective et le frayage au-delà de l'anneau de l'horizon est déplacé sur le plan terrestre, celui des champs des paysans, réalité économique indispensable au projet émancipateur. Réalité alimentaire - primordiale en ces temps de famine - ici finement entrelacée aux motifs infinis et exponentiels du sans-objet, révélant un paysage éminemment complexe. Ce dernier article ajoute une nouvelle touche de couleur vivante à ce portrait démesuré de l'artiste qui, à défaut de produire une politique per se, aura tracé, par des voies rapides de dépassement, un chemin vers ce qui se tient au-delà de la politique.

1 - Léo Strauss, Persécution et l'art d'écrire, P. : Gallimard, 2009.

2 - Tetyana Filevska, Kazimir Malévitch : la période kiévienne, 1928-1930, Kiev :

Rodovid, 2018.

\section{Emanuel Landolt}

Université de Saint-Gall, Suisse 